Article

\title{
Effects of Micromachining Processes on Electro-Osmotic Flow Mobility of Glass Surfaces
}

\author{
Yosuke Koga ${ }^{1}$, Reiko Kuriyama ${ }^{2}$, Yohei Sato ${ }^{2}$, Koichi Hishida ${ }^{2}$ and Norihisa Miki ${ }^{1, *}$ \\ 1 Department of Mechanical Engineering, Keio University, 3-14-1 Hiyoshi, Kohoku-ku, Yokohama, \\ Kanagawa, 223-8522, Japan; E-Mail: koga@a2.keio.jp \\ 2 Department of System Design Engineering, Keio University, 3-14-1 Hiyoshi, Kohoku-ku, \\ Yokohama, Kanagawa, 223-8522, Japan; E-Mails: kuriyama@tfe.sd.keio.ac.jp (R.K.); \\ yohei@sd.keio.ac.jp (Y.S.); hishida@sd.keio.ac.jp (K.H.) \\ * Author to whom correspondence should be addressed; E-Mail: miki@ mech.keio.ac.jp; \\ Tel.: +81-45-566-1430; Fax: +81-45-566-1495.
}

Received: 4 January 2013; in revised form: 16 February 2013 / Accepted: 20 February 2013 / Published: 13 March 2013

\begin{abstract}
Silica glass is frequently used as a device material for micro/nano fluidic devices due to its excellent properties, such as transparency and chemical resistance. Wet etching by hydrofluoric acid and dry etching by neutral loop discharge (NLD) plasma etching are currently used to micromachine glass to form micro/nano fluidic channels. Electro-osmotic flow (EOF) is one of the most effective methods to drive liquids into the channels. EOF mobility is affected by a property of the micromachined glass surfaces, which includes surface roughness that is determined by the manufacturing processes. In this paper, we investigate the effect of micromaching processes on the glass surface topography and the EOF mobility. We prepared glass surfaces by either wet etching or by NLD plasma etching, investigated the surface topography using atomic force microscopy, and attempted to correlate it with EOF generated in the micro-channels of the machined glass. Experiments revealed that the EOF mobility strongly depends on the surface roughness, and therefore upon the fabrication process used. A particularly strong dependency was observed when the surface roughness was on the order of the electric double layer thickness or below. We believe that the correlation described in this paper can be of great help in the design of micro/nano fluidic devices.
\end{abstract}

Keywords: surface roughness; electro-osmotic flow; glass; MicroTAS; nanofluidics; zeta potential; neutral loop discharge plasma etching; dry etching; wet etching 


\section{Introduction}

Because there are many beneficial size effects at the micro and nano scales, such as short mixing times, high efficiency in chemical reactions, and minute amounts of reagents and effluent liquids, micro/nano fluidic devices have been extensively studied for use in biomedical applications [1-20]. In particular, nano fluidic devices that contain nano channels can be used for separation and filtration [21], single-molecule detection [22-26], highly efficient PCR [27], chemical analysis [28,29] and nano-photonic sensors [30], with great help of development of nanofabrication technologies [31-43]. As the channels are reduced in size, the surface roughness and surface chemistry become dominant in determining the dynamics of flow. Electro-osmotic flow (EOF) is well-known to depend on the surface chemistry of the channel walls. An electric double-layer is formed near the channel wall, depending on the wall surface. When an external electric field is applied, the layer moves, generating EOF. Electroosmotic pumping is considered to be more efficient in driving liquids in micro/nano channels than applying differential pressure by an external pump, given the high viscous drag. The wall surface charge can be quantified by the zeta potential. Therefore, the EOF can be predicted by the given zeta potential and vice versa. The relationship between the zeta potential and EOF can be described by the Smoluchowski equation when the surface roughness is smaller than the electric double layer [44-46].

The EOF can be measured by micro-particle image velocimetry (PIV) [47-50]. Fluorescent nano/micro beads are typically used as tracers, and a confocal microscope can be used to deduce the velocity distribution in three dimensions [51-59]. Evanescent waves generated by total index reflection of a laser can be used to derive the velocity near the liquid/wall interface, within approximately $50 \mathrm{~nm}$, when they are used as the excitation light for the fluorescent tracer particles [60-64].

As we described above, the zeta potential can be calculated from a precisely measured EOF using the Smoluchowski equation, but this approach cannot be always applied to practical cases. The relation was modified for non-wetting charged surfaces by considering the fluid slip effect [65], which was experimentally validated [66]. Surface roughness is another factor that needs to be taken into consideration. Since surface roughness does affect EOF, this leads to errors in the zeta potential. On the other hand, even though the zeta potential of the channel wall material was known, it cannot accurately predict the resulting EOF in manufactured nano channels due to the surface roughness.

The surface roughness depends on the manufacturing process [67]. For example, glass, one of the most commonly used materials due to its superior chemical resistivity and transparency, can be patterned by wet etching using hydrofluoric acid (HF), dry etching, or mechanical machining. Wet etching is an isotropic technique but can be conducted at low cost. Dry etching can create anisotropic profiles with a high accuracy and a high aspect ratio. Mechanical machining can be used to fabricate three-dimensional structures. Each etching process culminates in a different surface geometry, depending on the etching conditions.

In this work, we attempted to experimentally obtain the EOF mobility, which is affected by both surface roughness and surface chemistry. The surface roughness is strongly affected by the manufacturing method and etching conditions. We prepared glass channels by wet etching and dry etching using various recipes, and formed micro-channels by attaching micropatterned polydimethyl siloxane (PDMS). We measured the EOF near the glass surface using microPIV, and the glass surface geometry was investigated by atomic force microscopy (AFM). We attempted to correlate the surface 
topography, and thus, the micromaching processes, with the EOF mobility. Since the Smoluchowski equation can be used to deduce the zeta potential only when the roughness is smaller than the electric double layer thickness, the EOF mobility obtained in the experiments cannot provide the true zeta potential that represents the chemical properties of the surface. Instead, we discuss the potential usage of an effective zeta potential, which is obtained by substituting the EOF mobility into the Smoluchowski equation and depends on both the chemical and topographical properties of the surface. When the effective zeta potential of micromachined glass surfaces is known, the EOF can be deduced using the Smoluchowski equation. The obtained data will be of great help in precisely designing highperformance nano/micro fluidic devices.

\section{Material and Method}

\subsection{Micromachining of Glass Substrates}

In our experiments, we used borosilicate glass (C030401, Matsunami Glass Ind., Ltd., Osaka, Japan) as the wall material. Hydrofluoric acid (HF) was purchased from Wako Pure Chemical Industries, Ltd., Osaka, Japan. Plasma etching of glass was conducted by NLD-570 (Ulvac, Inc., Kanagawa, Japan). The surface geometry after the machining process was investigated by atomic force microscopy (SPM-9700, Shimadzu, Kyoto, Japan).

The most frequently used glass manufacturing method is wet etching by HF. HF can pattern glass by the following reaction;

$$
\mathrm{SiO}_{2}+6 \mathrm{HF} \rightarrow \mathrm{H}_{2} \mathrm{SiF}_{6}+2 \mathrm{H}_{2} \mathrm{O}
$$

We conducted two experiments. First, we fixed the etching depth at $10 \mu \mathrm{m}$ and used HF with concentrations of $1 \%, 3 \%, 5 \%, 7 \%$, and $9 \%$. The required etching periods were 5460, 1500, 580, 330, and $280 \mathrm{~s}$, respectively. In the second experiment, glass substrates were immersed in $5 \% \mathrm{HF}$ for 5, 10, 15,20 , or $25 \mathrm{~min}$. The etching was conducted in a cleanroom environment at $25{ }^{\circ} \mathrm{C}$ and $40 \% \mathrm{Rh}$. We prepared five samples for each condition. Note that in these experiments, the whole surfaces were etched, i.e., no mask pattern was used.

While deep reactive ion etching of silicon has been extensively used in manufacturing MEMS for decades [68,69], the etching of glass using neutral loop discharge (NLD) is a promising technology for manufacturing glass micro/nano structures [70-73]. NLD employs plasma generated along a closed magnetic neutral line by a radio frequency (RF) electric field. NLD plasma has a high plasma density at a relatively low gas pressure, and can be applicable to high-aspect-ratio anisotropic etching.

In our experiments, borosilicate glass plates were etched by NLD-570 while they were attached to a 6 -inch silicon carbide wafer with a silicone oil compound to dissipate heat during the etching. We varied the antenna power to be $250,500,1200$, or $1800 \mathrm{~W}$. The etching periods were controlled to achieve 5- $\mu$ m-deep patterns, which led to 5880, 3840, 1800, or $1560 \mathrm{~s}$ etch times, respectively. Other representative etching parameters are: APC pressure of $0.4 \mathrm{~Pa}$, trigger pressure of $1 \mathrm{~Pa}$, PFC pressure of $400 \mathrm{~Pa}$, both gas flow rates of $\mathrm{C}_{3} \mathrm{~F}_{8}$ and $\mathrm{CHF}_{3}$ of $10 \mathrm{sccm}$, bias RF power of $300 \mathrm{~W}$, currents for magnet top, middle, and bottom of $12.2 \mathrm{~A}, 17 \mathrm{~A}, 12.2 \mathrm{~A}$, respectively. We prepared five samples for each condition. Note that in these experiments, the whole surfaces were etched, i.e., no mask pattern was used. 
The surface geometry was investigated by atomic force microscopy SPM-9700after the machining process. 10 measurement areas $10 \mu \mathrm{m}$ by $10 \mu \mathrm{m}$ in size were randomly selected from each sample.

\subsection{Measurement of EOF}

The EOF velocity was measured by micro PIV. When charged tracing particles are used for the measurement, the measured velocity is the summation of EOF and electrophoretic flow velocities [56]. The electrophoretic velocity under the identical measurement conditions was obtained in advance and then, subtracted from the measured velocity by micro PIV to deduce the EOF velocity.

First, as described above, glass plates were micromachined by either wet etching with HF or dry etching using NLD plasma. Note that these etching processes created no patterns on the glass surfaces. A polydimethyl siloxane (PDMS, Silpot 184, Toray, Tokyo, Japan) structure with a channel $1 \mathrm{~mm}$ in width, $30 \mathrm{~mm}$ in length, and $100 \mu \mathrm{m}$ in height and inlet/outlet holes molded from photolithography patterned SU-8 (SU-8 3000, Nippon Kayaku, Tokyo, Japan) was bonded onto the glass plates after oxygen plasma treatment, as shown in Figure 1. Fluorescent micro beads $0.2 \mu \mathrm{m}$ in diameter (FluoSpheres carboxylate-modified microspheres $0.2 \mu \mathrm{m}$ yellow-green fluorescent, Molecular Probes, Inc., Eugene, OR, USA) were used as the tracing particles. These beads were excited by a laser with a wavelength of $505 \mathrm{~nm}$, and emitted fluorescence at $515 \mathrm{~nm} .0 .5 \mathrm{~mL}$ of carboxylate solution containing $2 \mathrm{wt} \%$ of microbeads was mixed with $99.5 \mathrm{~mL}$ of deionized water. Then, this solution was mixed with a solution containing $1 \mathrm{Ml}$ of $1 \mathrm{M}$ HEPES (4-(2-hydroxyethyl)-1-piperazineethanesulfonic acid), $1 \mathrm{~mL}$ of $0.1 \mathrm{M} \mathrm{KCl}$, and $98 \mathrm{~mL}$ of deionized water. We introduced this $0.005 \%$ fluorescent micro-bead solution into the channel. An electric field of $13.3 \mathrm{~V} / \mathrm{mm}$ was applied and an EOF was generated. The experiments were conducted at $25{ }^{\circ} \mathrm{C}$ and $40 \% \mathrm{Rh}$. $\mathrm{pH}$ of the fluorescent solution was 7.15 and the produced electric double layer thickness was calculated to be $30.2 \mathrm{~nm}$ under these conditions. The velocity at the center of the channel in the horizontal plane and $5 \mu \mathrm{m}$ above the glass surface was measured by micro-PIV. Though three channel walls were made of PDMS, the EOF generated near the glass surface was determined by the characteristics of the glass surface [56]. In order to prevent electrolysis, the measurement started $2 \mathrm{~s}$ after the electric field was applied and the measurement time was limited to be $2 \mathrm{~s}$. Note that we experimentally confirmed that the EOF became steady $1 \mathrm{~s}$ after the field was applied. The micro PIV measurement was conducted one time for a sample since it was difficult to wash out all the tracing particles from the channel after each measurement, i.e., 5 EOF velocity data were obtained for one machining condition.

Figure 1. A micro-channel used to evaluate the electro-osmotic flow (EOF).

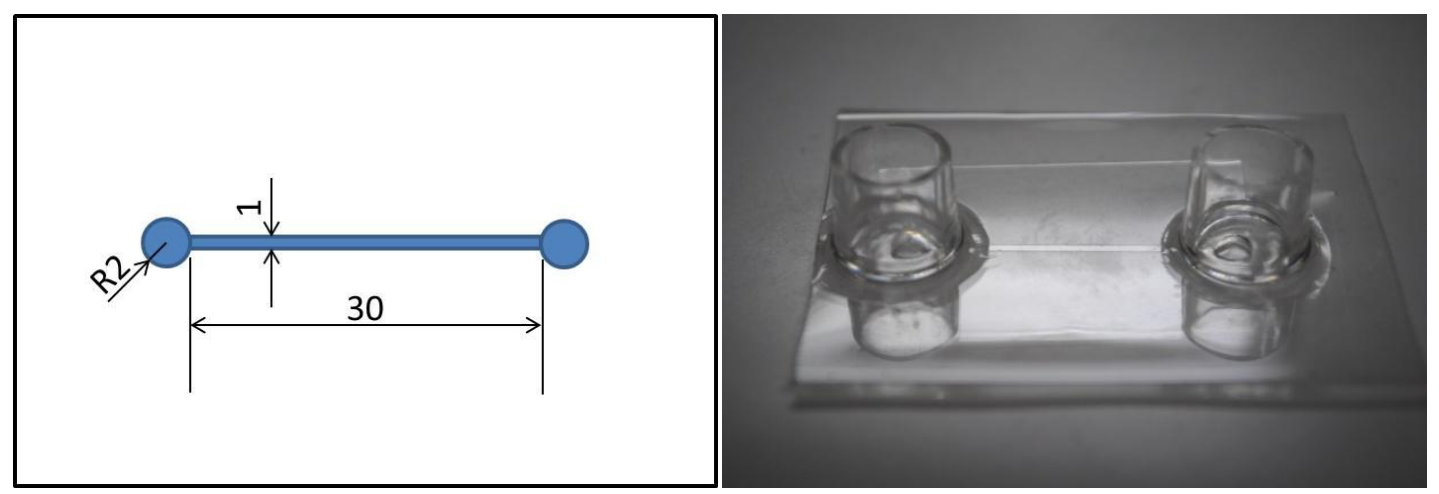




\section{Experiments}

\subsection{Surface Roughness after Glass Patterning}

Figure 2 shows surface topography images of untreated glass, glass wet-etched by $5 \mu \mathrm{m}$, and glass dry-etched by $5 \mu \mathrm{m}$ obtained by AFM. Dry etching using NLD plasma drastically increased the surface roughness, while wet etching did not. In wet etching using HF, the concentration of HF did not affect the surface roughness, as represented by the arithmetic mean value $R_{a}$ except the case of $1 \%$ concentration, as shown in Figure 3a. $R_{a}$ of the non-treated glass surface was $43 \mathrm{~nm}$, while that of the glass treated by $1 \% \mathrm{HF}$ was $61 \mathrm{~nm}$. This was most likely caused by the long wet etching time required by $1 \% \mathrm{HF}$ of $5460 \mathrm{~s}$ to achieve an etching depth of $10 \mu \mathrm{m}$. When $5 \% \mathrm{HF}$ was used as the etchant, the surface roughness decreased in the first 15 min of etching, which was a nature of isotropic wet etching. The surface roughness increased slightly when the etching time was greater than 20 min as shown in Figure $3 b$.

On the contrary, $R_{a}$ of the surface etched by NLD plasma strongly depended on the plasma power when we fixed the etching depth at $5 \mu \mathrm{m}$, as shown in Figure 4a. $R_{a}$ increased from approximately 160 $\mathrm{nm}$ to $280 \mathrm{~nm}$ when the plasma power was increased from $250 \mathrm{~W}$ to $1800 \mathrm{~W}$. For a plasma power of $1200 \mathrm{~W}, R_{a}$ increased from $40 \mathrm{~nm}$ to $210 \mathrm{~nm}$ as the etching progressed, as shown in Figure $4 \mathrm{~b}$.

\subsection{EOF Measurement}

The EOF velocity generated in channels with glass surfaces treated by wet etching is summarized in Figure 3. Figure 3a shows the surface roughness and EOF velocity for glass substrates etched by 10 $\mu \mathrm{m}$ using different concentrations of HF. The glass substrate treated by $1 \% \mathrm{HF}$ generated an EOF velocity of $660 \mu \mathrm{m} / \mathrm{s}$, while the non-treated glass substrate generated an EOF velocity of $870 \mu \mathrm{m} / \mathrm{s}$. Figure $3 \mathrm{~b}$ shows the relationship between the etching time by $5 \% \mathrm{HF}$ and the EOF velocity. In both graphs, the EOF velocity decreased with increasing surface roughness, and there was a strong correlation between surface roughness and EOF velocity.

For dry-etched samples, as shown in Figure 4, the EOF velocity decreased as the roughness increased, although the trend was not as strong as with the wet-etched samples. Figure 4a shows EOF velocities for glass substrates etched by $5 \mu \mathrm{m}$ with various antenna RF power values in NLD etching. The etching time was controlled to achieve a depth of $5 \mu \mathrm{m} .0 \mathrm{~W}$ corresponds to a non-treated glass substrate. The EOF velocity slightly decreased with increasing power, and was $500 \mu \mathrm{m} / \mathrm{s}$ at $1800 \mathrm{~W}$. The EOF velocity as a function of etching depth at $1200 \mathrm{~W}$ is shown in Figure $4 \mathrm{~b}$. The EOF velocity decreased with increasing time, and reached $510 \mu \mathrm{m} / \mathrm{s}$ at a depth of $5 \mu \mathrm{m}$. 
Figure 2. Atomic force microscopy (AFM) images of (a) non-treated, (b) wet-etched, and (c) dry-etched glass surfaces.

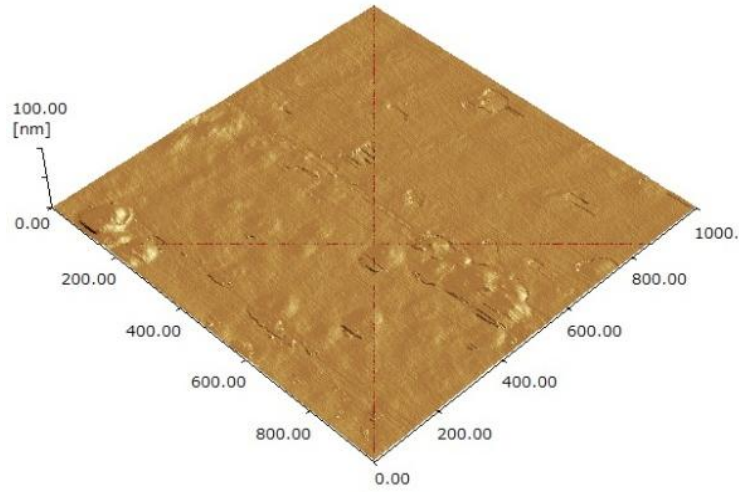

(a)

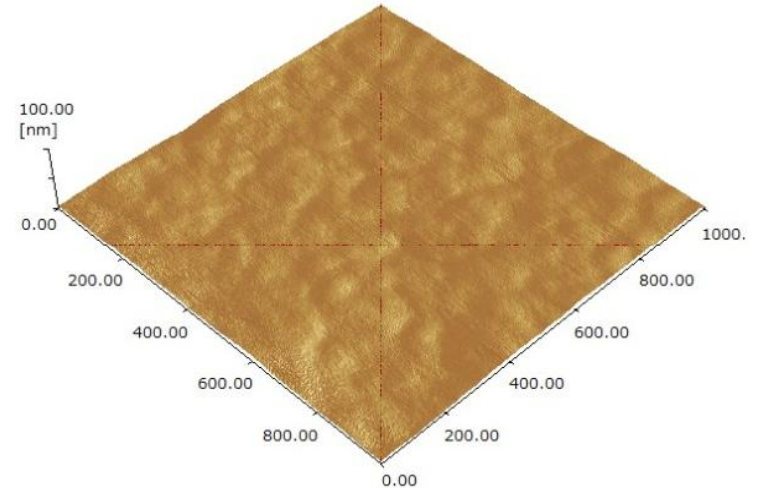

(b)

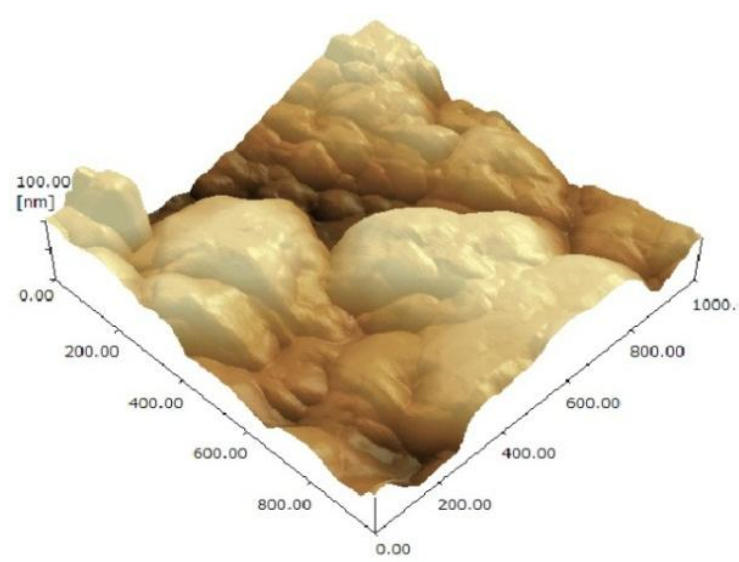

(c)

Figure 3. Surface roughness $\left(R_{a}\right)$ and the EOF velocity of glass surfaces treated by hydrofluoric acid (HF) (a) with different HF concentrations and (b) for different etching times.

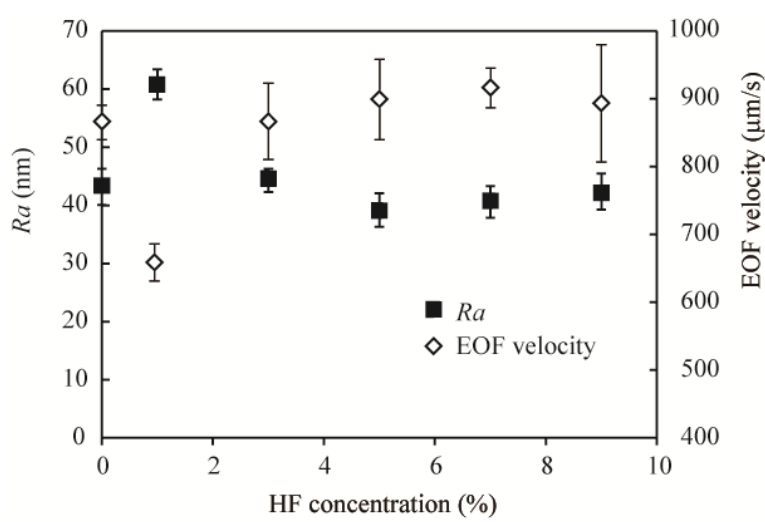

(a)

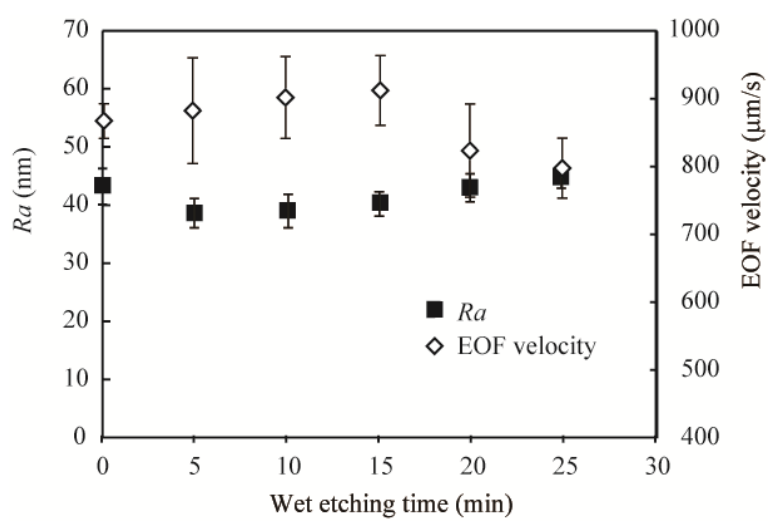

(b)

Figure 5 summarizes the relationship between the EOF velocity and the surface roughness $R_{a}$ of the samples used in the experiments. The white diamonds represent the wet-etched samples and the black diamonds represent the dry-etched samples. The graph indicates that the EOF velocity decreased drastically until the surface roughness reached $60 \mathrm{~nm}$. When the surface roughness exceeded $100 \mathrm{~nm}$, 
the EOF velocity decreased, but the trend was weak. The EOF strongly depends on the electric double layer, which is $30.2 \mathrm{~nm}$ at the ionic concentrations used in our experiments. Therefore, when the roughness is on the same order as the electric double layer, the EOF velocity is very sensitive to the roughness. When the roughness is several times greater than the electric double layer, the EOF is only slightly affected by the roughness.

Figure 4. Surface roughness $\left(R_{a}\right)$ and the EOF velocity for the glass surfaces treated by neutral loop discharge (NLD) plasma etching (a) with different plasma antenna power values and (b) for different etching depths with a plasma power of $1200 \mathrm{~W}$.

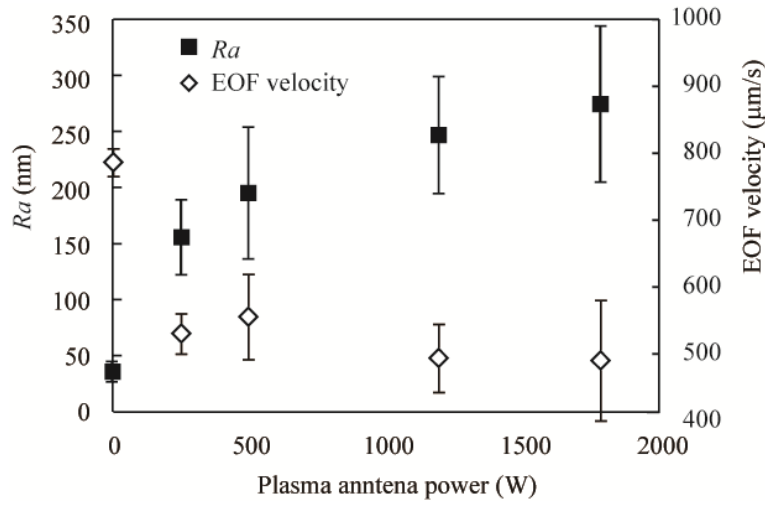

(a)

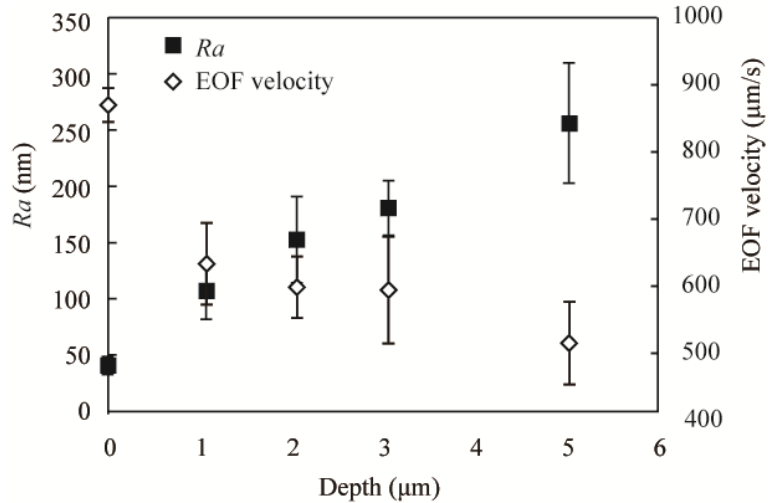

(b)

\subsection{Effective Zeta Potential}

The EOF velocity depends on the electric field, viscosity and permittivity of the liquid, and the surface properties of the channel. The surface properties include physical characteristics, surface roughness, and chemical properties. The zeta potential is used as a measure of the chemical properties, and can be deduced using the Smoluchowski equation if the roughness is smaller than the thickness of the electric double layer,

$$
U=\frac{\varepsilon \zeta}{\mu} E
$$

where $U$ is the average velocity in the channel, $\mu$ and $\varepsilon$ are the viscosity and permittivity, respectively, $\zeta$ is the zeta potential, and $E$ is the applied electric field strength [46].

Figure 5. EOF velocity as a function of surface roughness.

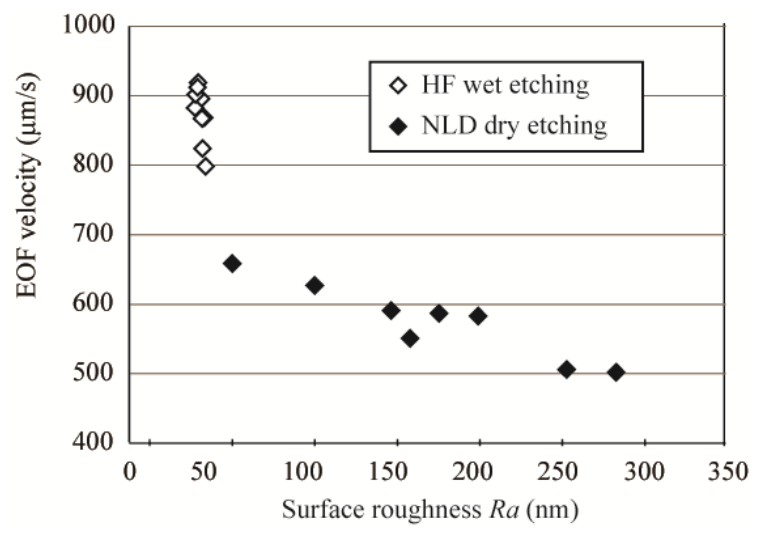


Figure 6. Effective zeta potential as a function of surface roughness.

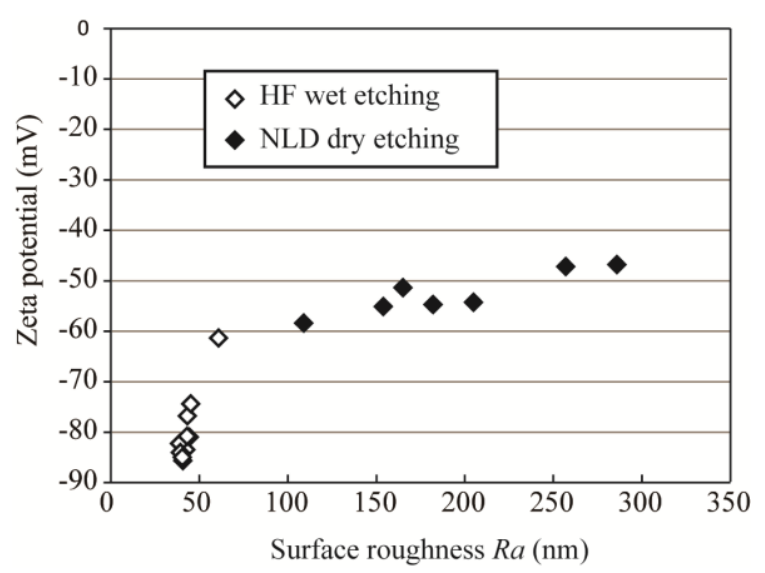

In our experiments, given the large surface roughness, Equation (2) cannot be used to deduce the true zeta potential that represents the chemical properties of the surface. However, Equation (2) will provide the effective zeta potential, which includes both the chemical properties and the topography of the surface. In designing nano/micro fluidic devices utilizing EOF, this effective zeta potential, which depends on the fabrication process and the surface roughness, should be used instead of the true zeta potential.

Figure 6 shows the effective zeta potential from Equation (2), substituting $U$ by the experimentally deduced average velocity in the channel, $\mu$ and $\varepsilon$ by $8.5 \times 10^{-4} \mathrm{~Pa} \cdot \mathrm{s}$ and $6.87 \times 10^{-10} \mathrm{~F} / \mathrm{m}$, respectively, and $E$ by $13.3 \mathrm{~V} / \mathrm{mm}$. The zeta potential ranged from $-86 \mathrm{mV}$ to $-47 \mathrm{mV}$ when $R_{a}$ varied from $43 \mathrm{~nm}$ to $290 \mathrm{~nm}$. In particular, when the roughness was on the same order as the electric double layer, the zeta potential was found to be sensitive to the roughness.

\section{Conclusions}

The glass manufacturing processes determines the surface roughness. Wet etching by HF produces minimal surface roughness and therefore, the EOF velocities do not vary from the non-treated glass, but it is isotropic and may not to be suitable for precision nanoscale manufacturing. NLD plasma dry etching is a promising anisotropic manufacturing process for nano/micro glass channels, although it was found to increase the surface roughness, adversely affecting the EOF velocities.

The EOF velocity showed a strong correlation with the surface roughness, particularly when the arithmetic mean value of $R_{a}$ was on the same order as the thickness of the electric double layer. Therefore, we proposed the use of an effective zeta potential that takes both chemical and physical properties of the surfaces into consideration. The relationship between the surface roughness or manufacturing processes, and EOF velocities or the effective zeta potential, obtained in this work can be readily applied to the precise design of nano/micro fluidic devices.

\section{Acknowledgements}

This work was supported by a Grant-in-Aid for Scientific Research (S) (21226006). We greatly appreciate the "Sensing center for nano/micro thermofluid \& thermal properties, Keio University" of 
"Low-Carbon Research Network" funded by the Ministry of Education, Culture, Sports, Science and Technology (MEXT), Japan for the microPIV system.

\section{References}

1. Manz, A.; Graver, N.; Widmer, H.M. Miniaturized total chemical-analysis systems-a novel concept for chemical sensing. Sens. Actuators B 1990, 1, 244-248.

2. Shoji, S. Fluids for sensor systems. Top. Curr. Chem. 1998, 194, 163-188.

3. Khandurina, J.; Guttman, A. Bioanalysis in microfluidic devices. J. Chromatogr. A 2002, 943, $159-183$.

4. Chovan, T.; Guttman, A. Microfabricated devices in biotechnology and biochemical processing. Trends Biotechnol. 2002, 20, 116-122.

5. Huikko, K.; Kostiainen, R.; Kotiaho, T. Introduction to micro-analytical systems: Bioanalytical and pharmaceutical applications. Eur. J. Pharm. Sci. 2003, 20, 149-171.

6. Sheehan, A.D.; Quinn, J.; Daly, S.; Dillon, P.; O'Kennedy, R. The development of novel miniaturized immuno-sensing devices: A review of a small technology with a large future. Anal. Lett. 2003, 36, 511-537.

7. Sato, K.; Hibara, A.; Tokeshi, M.; Hisamoto, H.; Kitamori, T. Microchip-based chemical and biochemical analysis systems. Adv. Drug Deliv. Rev. 2003, 55, 379-391.

8. Lee, S.J.; Lee, S.Y. Micro total analysis system (mu-TAS) in biotechnology. Appl. Microbiol. Biot. 2004, 64, 289-299.

9. Sun, Y.; Kwok, Y.C. Polymeric microfluidic system for DNA analysis. Anal. Chim. Acta 2006, 556, 80-96.

10. Gu, Y.; Miki, N. A microfilter utilizing a polyethersulfone porous membrane with nanopores. $J$. Micromech. Microeng. 2007, 17, 2308-2315

11. Tanaka, Y.; Sato, K.; Shimizu, T.; Yamato, M.; Okano, T.; Kitamori, T. Biological cells on microchips: New technologies and applications. Biosens. Bioelectron. 2007, 23, 449-458.

12. Chen, Y.; Wang, X.H.; Hong, M.; Erramilli, S.; Mohanty, P. Surface-modified silicon nanochannel for urea sensing. Sens. Actuators 2008, 133, 593-598.

13. Suzuki, H.; Takeuchi, S. Microtechnologies for membrane protein studies. Anal. Bioanal. Chem. 2008, 391, 2695-2702.

14. Gu, Y.; Miki, N. Multilayered microfilter using a nanoporous PES membrane and applicable as the dialyzer of a wearable artificial kidney. J. Micromech. Microeng. 2009, 19, doi:10.1088/ 0960-1317/19/6/065031.

15. Xu, X.; Zhang, S.; Chen, H.; Kong, J. Integration of electrochemistry in micro-total analysis systems for biochemical assays: Recent developments. Talanta 2009, 80, 8-18.

16. Ota, H.; Yamamoto, R.; Deguchi, K.; Tanaka, Y.; Kazoe, Y.; Sato, Y.; Miki, N. Three-dimensional spheroid-forming lab-on-a-chip using micro-rotational flow. Sens. Actuators B 2010, 147, 359-365.

17. Liu, K.K.; Wu, R.G.; Chuang, Y.J.; Khoo, H.S.; Huang, S-H.; Tseng, F-G. Microfluidic systems for biosensing. Sensors 2010, 10, 6623-6661.

18. Wlodkowic, D.; Cooper, J.M. Tumors on chips: Oncology meets microfluidics. Curr. Opin. Chem. Biol. 2010, 14, 556-567. 
19. Ota, H.; Kodama, T.; Miki, N. Rapid formation of size-controlled three dimensional hetero-cell aggregates using micro-rotation flow for spheroid study. Biomicrofluidics 2011, 5, doi:10.1063/1.3609969.

20. Ota, H.; Miki, N. Microfluidic experimental platform for producing size-controlled three-dimensional spheroids. Sens. Actuators A 2011, 169, 266-273.

21. Shen, C.; Mokkapati, V.R.S.S.; Pham, H.T.M.; Sarro, P.M. Micromachined nanofiltration modules for lab-on-a-chip applications. J. Micromech. Microeng. 2012, 22, doi:10.1088/0960-1317/22/2/025003.

22. Wang, K.; Yue, S.; Wang, L.; Jin, A.; Gu, C.; Wang, P.; Wang, H.; Xu, X.; Wang, Y.; Niu, H. Nanofluidic channels fabrication and manipulation of DNA molecules. IEEE Proc. Nanobiotechnol. 2006, 153, 11-15.

23. Das, S.K.; Austin, M.D.; Akana, M.C.; Deshpande, P.; Cao, H.; Xiao, M. Single molecule linear analysis of DNA in nano-channel labeled with sequence specific fluorescent probles. Nucleic Acids Res. 2010, 38, doi:10.1093/nar/gkq673.

24. Liu, C.; Qu, Y.Y.; Luo, Y.; Fang, N. Recent advances in single-molecule detection on micro- and nano-fluidic devices. Electrophoresis 2011, 32, 3308-3318.

25. Kish, L.L.; Kameoka, J.; Granqvist, C.G.; Kish, L.B. Log-normal distribution of single molecule fluorescence bursts in micro/nano-fluidic channels. Appl. Phys. Lett. 2011, 99, doi:10.1063/1.3648118.

26. Abad, E.; Juarros, A.; Retolaza, A.; Merino, S.; Marie, R.; Kristensen, A. DNA analysis by single molecule stretching in nanofluidic biochips. Microelectron. Eng. 2011, 88, 300-304.

27. Eun, D.S.; Shin, J.K.; Lee, J.H. Enhanced micro-PCR chip using TiO2nanofluidic heat-sink. Sens. Lett. 2009, 9, 276-282.

28. Chen, P.; Feng, X.; Du, W.; Liu, B-F. Microfluidic chips for cell sorting. Front. Biosci. 2008, 13, 2464-2483.

29. Mawatari, K.; Tsukahara, T.; Sugii, Y.; Kitamori, T. Extended-nano fluidic systems for analytical and chemical technologies. Nanoscale 2010, 2, 1588-1595.

30. Huang, M.; Yanik, A.A.; Chang, T.Y.; Altug, H. Sub-wavelength nanofluidics in photonic crystal sensors. Opt. Express 2009, 17, 24224-24233.

31. Cao, H.; Yu, Z.N.; Wang, J.; Tegenfeldt, J.O.; Austin, R.H.; Chen, E.; Wu, W.; Chou, S.Y. Fabrication of $10 \mathrm{~nm}$ enclosed nanofluidic channels. Appl. Phys. Lett. 2002, 81, 174-176

32. Pepin, A.; Youninou, P.; Studer, V.; Lebib, A.; Chen, Y. Nanoimprint lithography for the fabrication of DNA electrophoresis chips. Microelectron. Eng. 2002, 61, 927-932.

33. Zheng, H.J.; Wang, Z.L.; Feinerman, A.D. Fabrication of micro/nano fluidic channels with sacrificial galvanic coupled metals. Nanotechnology 2006, 17, 3183-3188.

34. Shao, P.E.; van Kan, A.; Wang, L.P.; Ansari, K.; Bettiol, A.A.; Watt, F. Fabrication of enclosed nanochannels in poly(methylmethacrylate) using proton beam writing and thermal bonding. Appl. Phys. Lett. 2006, 88, doi:10.1063/1.2181631.

35. Kehagias, N.; Chansin, G.; Reboud, V.; Zelsmann, M.; Schuster, C.; Kubenz, M.; Reuther, F.; Gruetzner, G.; Sotomayer Torres, C.M. Embedded nano channels fabricated by non-selective reverse contact UV nanoimprint lithography technique. Microelectron. Eng. 2007, 84, 921-924.

36. Wong, C.C.; Agarwal, A.; Balasubramanian, N.; Kwong, D.L. Fabrication of self-sealed circular nano/microfluidic channels in glass substrates. Nanotechnology 2007, 18, doi:10.1088/0957-4484/ 18/13/135304. 
37. Koller, D.M.; Galler, N.; Ditlbacher, H.; Hohenau, A.; Leitner, A.; Aussenegg, F.R.; Kren, J.R. Direct fabrication of micro/nano fluidic channels by electron beam lithography. Microelectron. Eng. 2009, 86, 1314-1316.

38. Yang, R.; Lu, B.R.; Wang, J.; Xie, S-Q.; Chen, Y.; Hug, E.; Qu, X-P.; Liu, R. Fabrication of micro/nano fluidic channels by nanoimprint lithography and bonding using SU-8. Microelectron. Eng. 2009, 86, 1379-1381.

39. Venkatakrishnan, K.; Jariwala, S.; Tan, B. Maskless fabrication of nano-fluidic channels by two-photon absorption (TPA) polymerization of SU-8 on glass substrate. Opt. Express 2009, 17, 2756-2762.

40. Kim, S.K.; Cho, H.; Park, H.K.; Kim, J.H.; Chung, B.H. Fabrication of nanochannels by anisotropic wet etching on silicon-on-insulator wafers and their applications to DNA stretch. $J$. Nanosci. Nanotechnol. 2010, 10, 637-642.

41. Fernandez-Cuesta, I.; Palmarelli, A.L.; Liang, X.G.; Zhang, J.; Dhuey, S.; Olynick, D.; Cabrini, S. Fabrication of fluidic devices with $30 \mathrm{~nm}$ nanochannels by direct imprinting. J. Vac. Sci. Technol. B 2011, 29, doi:10.1116/1.3662886.

42. Afanasiev, A.; Lahdesmaki, I.; Parviz, B.A. Fabrication and electrical characterization of integrated nano-scale fluidic channels. Microsyst. Technol. 2011, 17, 1511-1518.

43. Hong, L.F.; Pan, T.R. Surface microfluidics fabricated by photopatternable superhydrophobic nanocomposite. Microfluid. Nanofluid. 2011, 10, 991-997.

44. Hunter, R.J. Zeta potential in Colloid Science: Principles and Applications; Academic Press: New York, NY, US, 1981.

45. Sze, A.; Erickson, D.; Ren, L. Zeta-potential measurement using the Smoluchowski equation and the slope of the current-time relationship in electrosmotic flow. J. Colloid Interf. Sci. 2003, 261, 402-410.

46. Venditti, R.; Xuan, X.C.; Li, D.Q. Experimental characterization of the temperature dependence of zeta potential and its effect on electroosmotic flow velocity in microchannels. Microfluid. Nanofluid. 2006, 2, 493-499.

47. Santiago, J.G.; Wereley, S.T.; Meinhart, C.D.; Beebe, D.J.; Adrian, R.J. A particle image velocimetry system for microfluidics. Exp. Fluids 1998, 25, 316-319.

48. Sato, Y.; Hishida, K. Electrokinetic effects on motion of submicron particles in microchannel. Fluid Dyn. Res. 2006, 38, 787-802.

49. Lindken, R.; Rossi, M.; Grosse, S.; Westerweel, J. Micro-Particle Image Velocimetry (mu PIV): Recent developments, applications, and guidelines. Lab Chip 2009, 9, 2551-2567.

50. Tatsumi, K.; Nishitani, K.; Fukuda, K.; Katsumoto, Y.; Nakabe, K. Measurement of electroosmotic flow velocity and electric field in microchannels by micro-particle image velocimetry. Meas. Sci. Technol. 2010, 21, doi:10.1088/0957-0233/21/10/105402.

51. Park, J.S.; Choi, C.K.; Kihm, K.D. Optically sliced micro-PIV using confocal laser scanning microscopy (CLSM). Exp. Fluids 2004, 37, 105-119.

52. Lima, R.; Wada, S.; Tsubota, K.; Yamaguchi, T. Confocal micro-PIV measurements of threedimensional profiles of cell suspension flow in a square microchannel. Meas. Sci. Technol. 2006, 17, 797-808.

53. Park, J.S.; Kihm, K.D. Use of confocal laser scanning microscopy (CLSM) for depthwise resolved microscale-particle image velocimetry ( $\mu$-PIV). Opt. Lasers Eng. 2006, 44, 208-223. 
54. Ichiyanagi, M.; Sato, Y.; Hishida, K. Optically sliced measurement of velocity and $\mathrm{pH}$ distribution in microchannel. Exp. Fluids 2007, 43, 425-435.

55. Kinoshita, H.; Kaneda, S.; Fujii, T.; Oshima, M. Three-dimensional measurement and visualization of internal flow of a moving droplet using confocal micro-PIV. Lab Chip 2007, 7, $338-346$.

56. Ichiyanagi, M.; Sasaki, S.; Sato, Y.; Hishida, K. Micro-PIV/LIF measurements on electrokinetically-driven flow in surface modified microchannels. J. Micromech. Microeng. 2009, 19, doi:10.1088/0960-1317/19/4/045021.

57. Lee, S.J.; Kim, S. Advanced particle-based velocimetry techniques for microscale flows. Microfluid. Nanofluid. 2009, 6, 577-588.

58. Oishi, M.; Kinoshita, H.; Fujii, T.; Oshima, M.. Simultaneous measurement of internal and surrounding flows of a moving droplet using multicolour confocal micro-particle image velocimetry (micro-PIV). Meas. Sci. Technol. 2011, 22, doi:10.1088/0957-0233/22/10/105401.

59. Williams, S.J.; Park, C.; Wereley, S.T. Advances and applications on microfluidic velocimetry techniques. Microfluid. Nanofluid. 2010, 8, 709-726.

60. Zettner, C.M.; Yoda, M. Particle velocity field measurements in a near-wall flow using evanescent wave illumination. Exp. Fluids 2003, 34, 115-121.

61. Banerjee, A.; Kihm, K.D. Three-dimensional tracking of nanoparticles using R-TIRFM technique. J. Heat Trans. 2004, 126, doi:10.1115/1.1811724.

62. Kazoe, Y.; Sato, Y. Effect of ion motion on zeta-potential distribution at microchannel wall obtained from nanoscale laser-induced fluorescence. Anal. Chem. 2007, 79, 6727-6733.

63. Kazoe, Y.; Miyakawa, S.; Miki, N.; Sato, Y. Fluorescence imaging technique of surface electrostatic potential using evanescent wave illumination. Appl. Phys. Lett. 2009, 95, doi:10.1063/1.3266842.

64. Min, Y.U.; Kim, K.C. Hybrid micro-/nano-particle image velocimetry for 3D3C multi-scale velocity field measurement in microfluidics. Meas. Sci. Technol. 2011, 22, doi:10.1088/ 0957-0233/22/6/064001.

65. Joly, L.; Ybert, C.; Trizac, E.; Bocquet, L. Liquid friction on charged surfaces: From hydrodynamic slippage to electrokinetics. J. Chem. Phys. 2006, 125, doi:10.1063/1.2397677.

66. Lim, J.M.; Chun, M.S. Curvature-induced secondary microflow motion in steady electro-osmotic transport with hydrodynamic slippage effect. Phys. Fluids 2011, 23, doi:10.1063/1.3650911.

67. Miki, N.; Spearing, S.M. Effect of nanoscale surface roughness on the bonding energy of direct-bonded silicon wafers. J. Appl. Phys. 2003, 94, 6800-6806.

68. Ayón, A.A.; Braff, R.; Lin, C.C., Sawin, H.H.; Schmidt, M.A. Characterization of a time multiplexed inductively coupled plasma etcher. J. Electrochem. Soc. 1999, 146, 339-349.

69. Miki, N.; Teo, C.J.; Ho, L.C.; Zhang, X. Enhancement of rotordynamic performance of high-speed micro-rotors for power MEMS applications by precision deep reactive ion etching. Sens. Actuators A 2003, 104, 263-267.

70. Chen, W.; Itoh, M.; Hayashi, T.; Uchida, T. Dry etch process in magnetic neutral loop discharge plasma. Jpn. J. Appl. Phys. 1998, 37, 332-336.

71. Chen, W.; Morikawa, Y.; Itoh, M.; Hayashi, T.; Sugita, K.; Shindo, H.; Uchida, T. Very uniform and high aspect ratio anisotropy $\mathrm{SiO} 2$ etching process in magnetic neutral loop discharge plasma. 
J. Vac. Sci. Technol. A 1999, 17, 2546-2550.

72. Chen, W.; Sugita, K.; Morikawa, Y.; Yasunami, S.; Hayashi, T.; Uchida, T. Application of magnetic neutral loop discharge plasma in deep silica etching. J. Vac. Sci. Technol. A 2001, 19, 2936-2940.

73. Uchida, T.; Hamaguchi, S. Magnetic neutral loop discharge (NLD) plasmas for surface processing. J. Phys. D 2008, 41, doi:10.1088/0022-3727/41/8/083001.

(C) 2013 by the authors; licensee MDPI, Basel, Switzerland. This article is an open access article distributed under the terms and conditions of the Creative Commons Attribution license (http://creativecommons.org/licenses/by/3.0/). 